\title{
ASYMPTOTIC SOLUTION OF A MODEL FOR BILAYER ORGANIC DIODES AND SOLAR CELLS*
}

\author{
GILES RICHARDSON ${ }^{\dagger}$, COLIN PLEASE $^{\dagger}, \mathrm{JAMIE} \mathrm{FOSTER}^{\dagger}$, AND JAMES KIRKPATRICK ${ }^{\ddagger}$
}

\begin{abstract}
Organic diodes and solar cells are constructed by placing together two organic semiconducting materials with dissimilar electron affinities and ionization potentials. The electrical behavior of such devices has been successfully modeled numerically using conventional drift diffusion together with recombination (which is usually assumed to be bimolecular) and thermal generation. Here a particular model is considered and the dark current-voltage curve and the spatial structure of the solution across the device is extracted analytically using asymptotic methods. We concentrate on the case of Shockley-Read-Hall recombination but note the extension to other recombination mechanisms. We find that there are three regimes of behavior, dependent on the total current. For small currents - i.e., at reverse bias or moderate forward bias - the structure of the solution is independent of the total current. For large currents-i.e., at strong forward bias - the current varies linearly with the voltage and is primarily controlled by drift of charges in the organic layers. There is then a narrow range of currents where the behavior undergoes a transition between the two regimes. The magnitude of the parameter that quantifies the interfacial recombination rate is critical in determining where the transition occurs. The extension of the theory to organic solar cells generating current under illumination is discussed as is the analogous current-voltage curves derived where the photo current is small. Finally, by comparing the analytic results to real experimental data, we show how the model parameters can be extracted from the shape of current-voltage curves measured in the dark.
\end{abstract}

Key words. Shockley model, drift diffusion, asymptotic analysis, photovoltaic

AMS subject classifications. 82D $37,34 \mathrm{E} 05,35 \mathrm{~B} 40,78 \mathrm{~A} 57$

DOI. $10.1137 / 110825807$

1. Introduction. Organic materials are promising materials for many semiconducting applications, for example, light-emitting diodes [9], field effect transistors [26], sensors [25], and solar cells [10]. They are already widely applied in the xerographic industry [6]. Their attraction is that they enable devices to be designed with similar properties to those made from traditional semiconductors, such as silicon and gallium arsenide, but at a fraction of the cost. This is primarily due to high throughput processing techniques such as roll-to-roll printing and screen printing of organic inks. A timely and exciting application of this technology, which is the main motivator for this paper, is that such devices provide the possibility of very cheap large-scale solar cells. There are many companies inching closer to making organic photovoltaic diodes with energy efficiencies sufficient for commercialization [21, 22].

A solar cell converts solar energy into electrical power by (a) converting photons into excited charges, (b) separating these charges, and (c) transporting them to an external circuit. Under operation, positive charges flow in one direction and negative

*Received by the editors February 25, 2011; accepted for publication (in revised form) September 18, 2012; published electronically November 15, 2012.

http://www.siam.org/journals/siap/72-6/82580.html

†School of Mathematics, University of Southampton, Southampton SO17 1BJ, UK (g.richardson@ soton.ac.uk, c.p.please@soton.ac.uk, j.m.foster@soton.ac.uk). The second author's work was supported by award KUK-C1-013-04, made by King Abdullah University of Science and Technology, and by the EPSRC through grant EP/I01702X/1. The first and third authors' work was supported by the EPSRC through grant EP/I01702X/1.

$\ddagger$ OCCAM, University of Oxford, 24-29 St Giles’ Oxford, OX1 3LB, UK (kirkpatrick@maths. ox.ac.uk). This author's work was supported by award KUK-C1-013-04, made by King Abdullah University of Science and Technology, and a James Martin fellowship. 
ones in the other. This inherent asymmetry is exactly the reason why photovoltaic devices act as diodes, even in the dark. Therefore understanding the current voltage characteristics of a solar cell in the dark is essential to explaining its behavior under illumination.

In an inorganic solar cell, the asymmetry is achieved by doping [23, 17]. In contrast, in an organic solar cell the asymmetry is created by using two different materials with different affinities for charge: one material, known as the acceptor, supports negative charges (free electrons), whereas the other, known as the donor, supports positive ones (holes). The material properties which govern this behavior are called the ionization potential (for donors) and the electron affinity (for acceptors).

A significant problem that influences the design of most organic solar cells is that when light is absorbed it leads to the formation of an excited state consisting of a bound charge pair (termed an exciton) which must reach the interface between the materials in order to separate into a hole and an electron, thereby releasing its energy as a usable electric current. However, excitons have a short lifetime. As a result they are only able to travel a short distance before they recombine and their energy is lost from the device. This distance is known as the exciton diffusion length and is typically around $10 \mathrm{~nm}$ [10]. The material used in organic solar cells must be around $200 \mathrm{~nm}$ [10] thick in order to absorb a large proportion of the solar spectrum. The existing solution to this problem is to make solar cells $200 \mathrm{~nm}$ thick and to create an interface between the two materials that is convoluted on the length scale of the exciton diffusion length. Many theoretical studies $[1,3,16]$ have therefore concentrated on the crucial role of this convoluted morphology to determine efficiency but of necessity have required numerical approaches in order to account for the complex geometry in the models. Another approach has been to use a homogenized model where the intimate mixture of the two physical materials is replaced by an effective medium $[8,11,15]$.

We believe that analytical results relevant to these technologies would be valuable. In order to properly account for complex morphology, it is essential to understand the behavior of a simpler device with a known and well characterized morphology. We therefore concentrate on bilayer devices where the device consists of two uniform layers of material so that the device is planar and the interface is flat. Such bilayer organic devices have been studied experimentally for example by $[4,20]$ and also modeled numerically by authors including $[1,2,5,7]$.

The general approach to modeling these devices is to consider the steady state and to treat charge transport in the donor and acceptor using a drift diffusion model. Physically recombination and generation of charges is taken to occur in a layer at the interface on a length scale compared to the size of molecules $(1 \mathrm{~nm})$. Two approaches are taken to account for this layer. In the first approach a special "mixed" layer is assumed to exist at the interface. In this layer the current continuity equations include generation and recombination. Different authors use different internal boundary conditions to account for the change in material properties. For example, Barker, Ramsdale, and Greenham [1] exclude electrons and holes from crossing the interface. Some authors account for jumps in the electric field due to dipole effects. In [2] Brinkman et al. adopt an approach in this vein. Brinkman's approach eliminates the sharp interface by smoothly varying ionization potential and electron affinity in the narrow interface layer. The second approach is to have a discontinuity in charge fluxes and denisities. This approach has not yet receieved much attention, although we note the work in [5], which also models a device with a sharp interface between two materials. In this paper we adopt the latter approach. We show that the jump in charge densities can be related to the jump in ionization potential and electron affin- 
ity. We also argue that the current flowing through the interface must be determined by a local law at the interface that models thermal generation of, and bimolecular recombination between, electrons and holes; this is in contrast to the model formulated in [5] in which bimolecular recombination is assumed to occur within the bulk but not at the interface and where the interface hole/electron current depends solely on the local hole/electron concentration at the interface and is controlled by thermionic emission over an energy barrier. We deduce the general form that this recombination/generation law must obey to ensure that the solution is compatible with detailed balance. We also argue that the jump in ionization potential and electron affinity across the interface is so large that it is reasonable to neglect the free electron concentration in the donor and the hole concentration in the acceptor. We then analyze the problem asymptotically in the physically relevant regime where the nondimensional parameter $\delta$ (which gives a measure of the ratio of the recombination rate to the diffusion rate) is small. Three distinct regimes for the resulting current flow are identified and analyzed. The first is for small currents (in reverse bias or in moderate forward bias) where the current-voltage curve is similar to that of a diode and the current varies exponentially with voltage [23]. The second is for large currents (in hard forward bias) where the current-voltage relationship is linear. The third is a narrow range of medium-sized currents where the behavior changes character smoothly between the first and the third. Asymptotic solutions for the small and large current regimes are derived analytically, while the intermediate current regime is shown to be characterized by an exact solution to the model whose coefficients are found by numerical solution of a set of transcendental equations. By comparing our analytical solutions to experimental data we show that it is possible to fit the behavior of the device at low and high voltages using the same model. Physically this result is significant because it indicates that the series resistance in bilayer cells is dominated by the resistivity of the materials themselves, whereas the diode behavior is dominated by recombination at the interface.

2. Equations. The main electrical processes taking place in an organic diode are charge transport in the bulk of both donor and acceptor materials and charge generation and recombination at the interface between the two. Before formulating a model for these processes it is helpful to review the physical mechanisms of electrical conduction in a semiconductor. In a conventional inorganic semiconducting material (such as silicon) the electrons have energies that predominantly lie in the valence band (low energy) and as such are immobile, behaving like electrons in an insulator. Above the valence band and separated from it by an appreciable energy gap there is a conduction band into which some electrons may jump if they possess sufficient thermal energy. Not only are electrons in the conduction band able to conduct electricity (and freely diffuse), but they leave a vacancy in the valence band (with net positive charge) termed a hole, which is also mobile and so contributes to the net electrical conductivity of the material. The energy of an electron at the lower edge of the conduction band $\psi_{n}$ and of a hole at the upper edge of the valence band $-\psi_{p}$ are not in general spatially independent, being affected by changes in material properties and electric fields. The spatial dependence of the energy levels is illustrated in a band diagram, such as that shown in Figure 2.1, and leads to a force on an electron $-\nabla \psi_{n}$ and on a hole $\nabla \psi_{p}$ which, when balanced with a "viscous drag," gives rise to electron and hole velocities down gradients in $\psi_{n}$ and up gradients in $\psi_{p}$, respectively. In addition, random thermal excitations lead to diffusive components of the motion for both particles. In organic semiconductors the picture is slightly more complicated because both electrons and holes typically lie in shallow (but highly localized) energy 


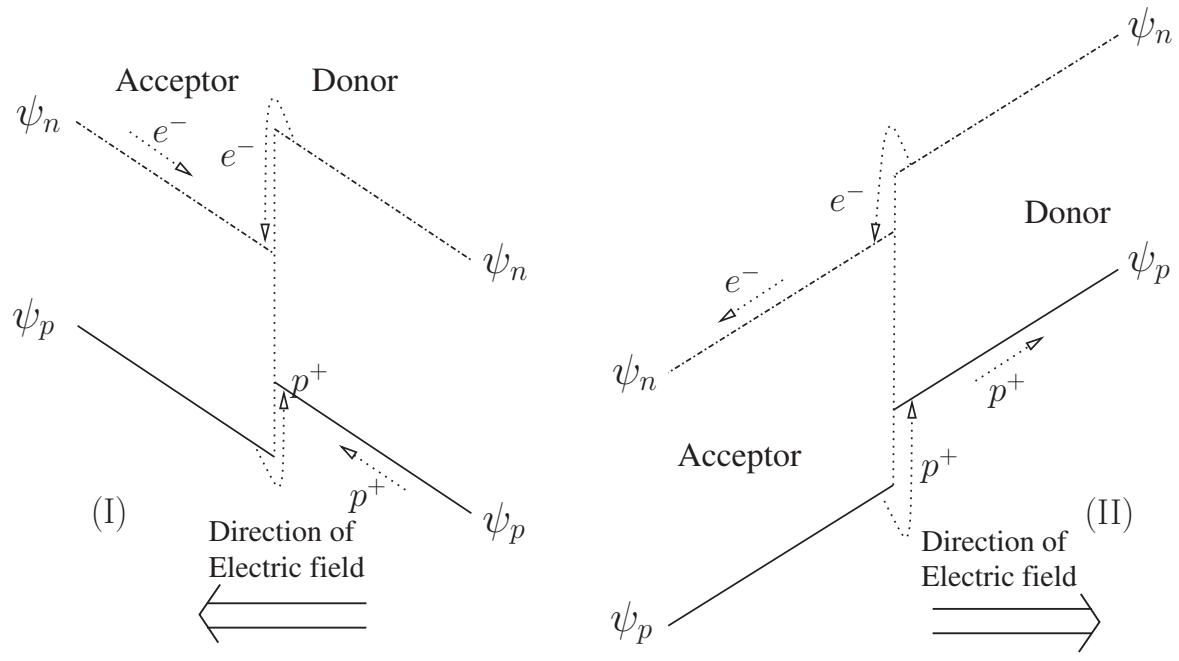

FIG. 2.1. Sketches of the band diagram for organic diodes in (I) forward bias and (II) reverse bias showing the energy levels of the HOMO by solid lines and the LUMO edge by dot-dashed lines. Arrows indicate the directions of the forces on electrons and holes due to variations in their electrochemical potential. At the semiconductor interface the jump in energy levels leads to migration of electrons from the donor to the acceptor and migrations of holes in the reverse direction. In forward bias the electric field tilts the LUMO and HOMO levels so that electrons flow toward the interface in the acceptor and holes flow toward the interface in the donor; this leads to high concentrations of these two species at the interface and to a high recombination current. In contrast, in reverse bias, the electric field tilts the LUMO and HOMO levels the other way so that electrons in the acceptor and holes in the donor migrate away from the interface, thus limiting interface recombination and ensuring that the current flowing in the device is primarily determined by generation on the interface. Since recombination of the majority carriers (electons in the acceptor and holes in the donor) at the interface typically controls current flow in the diode, much higher current flows can be achieved in forward bias than in reverse bias.

wells, termed traps, and move by hopping from one trap to the next. In part because of this difference in physics, it is unusual to refer to conduction and valence bands in organic semiconductors. It is much more common (though far from universal) to refer to the lowest unoccupied molecular orbital (LUMO), which plays a role analogous to the conduction band in inorganic materials, and to the highest occupied molecular orbital (HOMO), which plays a role analogous to the valence band. The importance of trapping in organic materials has led to the formulation of a variety of complex models for charge transport in organic semiconductors, including multiple trapping models [12], Gaussian disorder models [18], and atomistic models [14]. In this work (as in many others treating organic devices $[2,3,5,7,11]$ ) the motion of electrons and holes through the organic semiconductors is modeled by simple drift diffusion, allowing for the possibility that the diffusion coefficient depends upon electric field, while accounting for the differences in electron affinities and ionization potentials of the materials by letting these quantities be spatially dependent (see, for example, [23]). In the bilayer device that we consider here both electron affinity and ionization potential are piecewise constant with a sharp jump at the acceptor-donor interface. This jump manifests itself as a jump in the energy levels of the band edges $\psi_{n}$ and $\psi_{p}$ (as illustrated in Figure 2.1) and leads to the marked preference of the holes for the donor (high $\psi_{p}$ ) and of electrons for the acceptor (low $\psi_{n}$ ). Indeed, in a typical bilayer solar cell, the jumps in energy are so large that the concentrations of holes in the acceptor and electrons 


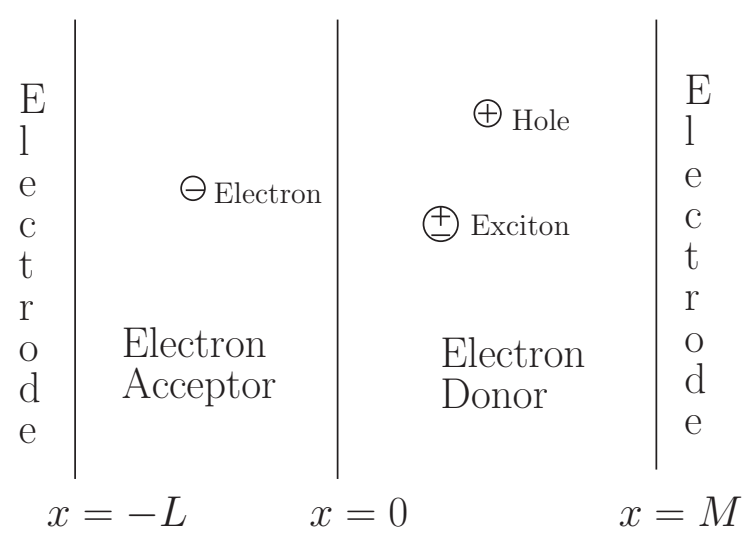

FIG. 2.2. The geometry of the solar cell.

in the donor are vanishingly small which is an important factor in limiting (undesirable) recombination of charge carriers in the bulk. The jumps in electron affinity and ionization potential also serve to dissociate excitons (generated by photo-absorption in the semiconductors) into free electrons in the acceptor and free holes in the donor.

The primary mechanisms by which electric current passes from the acceptor (where it is predominantly carried by electrons) through the interface into the donor (where it is predominantly carried by holes) are believed, in photovoltaic devices, to be the generation and recombination of electron-hole pairs $[3,11,15]$. The latter mechanism, which predominates where the current passes from the acceptor to the donor, results either from thermal emission (of electron-hole pairs) or dissociation of solar generated excitons (into electron-hole pairs). The former mechanism predominates where current passes from the donor to the acceptor and is a result of bimolecular recombination on the interface between electrons in the acceptor and holes in the donor.

A schematic for a simple bilayer organic photovoltaic device lying between $-L<$ $x<M$ with the interface at $x=0$ between the electron acceptor material $(-L<x<$ $0)$ and donor material $(0<x<M)$ is shown in Figure 2.2.

2.1. Current continuity equations in each material. In line with the discussion above we model the device by drift diffusion equations for electrons and holes coupled to Poisson's equation for the electric potential. We include the possibility of a spatially dependent electron affinity and ionization potential to model the preference of the donor material for holes and the acceptor for electrons but neglect any bulk recombination between holes and electrons, since we assume recombination primarily occurs on the acceptor-donor interface. The resulting one-dimensional equations take the form

$$
\begin{aligned}
\frac{\partial n}{\partial t}+\frac{\partial \mathcal{F}_{n}}{\partial x} & =0, \\
\frac{\partial p}{\partial t}+\frac{\partial \mathcal{F}_{p}}{\partial x} & =0, \\
\frac{\partial}{\partial x}\left(\varepsilon \frac{\partial \phi}{\partial x}\right) & =q(n-p), \\
\mathcal{F}_{n} & =-D_{n}\left(\frac{\partial n}{\partial x}+\frac{1}{k T} n \frac{\partial \psi_{n}}{\partial x}\right),
\end{aligned}
$$




$$
\begin{aligned}
\mathcal{F}_{p} & =-D_{p}\left(\frac{\partial p}{\partial x}-\frac{1}{k T} p \frac{\partial \psi_{p}}{\partial x}\right), \\
\psi_{n} & =\mu_{n}(x)-q \phi, \\
\psi_{p} & =\mu_{p}(x)-q \phi,
\end{aligned}
$$

where $\mathcal{F}_{n}$ is the flux of electrons, $\mathcal{F}_{p}$ is the flux of holes, $n$ is the charge density of electrons, $p$ is the charge density of holes, and $\phi$ is the electric potential. The material parameters in the problem are $\mu_{p}$ the ionization potential, $-\mu_{n}$ the electron affinity, $D_{n}$ the free electron diffusivity, $D_{p}$ the hole diffusivity, $k$ Boltzmann's constant, $T$ the absolute temperature, $\varepsilon$ the permittivity, and $q$ the charge on an electron. We shall term the functions $\psi_{n}$ and $\psi_{p}$ the energy levels of the LUMO and HOMO, respectively, while noting that there is some dispute about the correct notation for these quantities with some authors applying them to $\mu_{n}$ and $\mu_{p}$ instead. ${ }^{1}$ Electrons in the LUMO experience a force down gradients in $\psi_{n}$ while holes in the HOMO experience a force up gradients in $\psi_{p}$. At the interface the jumps in $\psi_{n}$ and $\psi_{p}$ (resulting from the discontinuities in $\mu_{n}$ and $\mu_{p}$ there) mean that electrons are attracted into the acceptor from the donor while holes experience a force in the opposite direction. Rough sketches of the band diagram of an organic diode in both forward and reverse bias are made in Figure 2.1. Note that the total current density $J$ can be expressed as the difference of hole and electron fluxes as follows:

$$
J=q\left(\mathcal{F}_{p}-\mathcal{F}_{n}\right) .
$$

In inorganic materials the diffusion coefficients are usually taken as constant or as functions of the material and temperature. However, unlike the movement of free charges in an inorganic semiconductor, transport in an organic material is primarily through charges jumping between nearby trap states. There is considerable interest in modeling this transport process, and one of the simplest descriptions is provided by the Poole-Frenkel model $[23,1,3]$, where the diffusivities $D_{n}$ and $D_{p}$ are taken to be functions of the local electric field. The form taken is usually

$$
D_{n}\left(\frac{\partial \phi}{\partial x}\right)=D_{n, 0} \exp \left(\gamma\left|\frac{\partial \phi}{\partial x}\right|^{1 / 2}\right), \quad D_{p}\left(\frac{\partial \phi}{\partial x}\right)=D_{p, 0} \exp \left(\gamma\left|\frac{\partial \phi}{\partial x}\right|^{1 / 2}\right),
$$

where $D_{n, 0}, D_{p, 0}$, and $\gamma$ are constants. More complex models can be derived accounting, for example, for a density of trap states in the material. Such a model results in the fraction of charges which are free to move being dependent on the charge density itself [12], so that the diffusivity depends directly on the charge density [24]. We do not include such effects here noting that the error made in approximating $D_{n}$ and $D_{p}$ by constants only becomes appreciable at very high fields.

The model above allows for general structures in the device, but here we are interested in modeling two adjacent regions, each of a uniform material; therefore we assume that the electron affinity and ionization potential are constant in each material so that

$$
\mu_{n}=\left\{\begin{array}{ll}
\mu_{n-} & \text { in } x<0, \\
\mu_{n+} & \text { in } x>0,
\end{array} \quad \mu_{p}= \begin{cases}\mu_{p-} & \text { in } x<0, \\
\mu_{p+} & \text { in } x>0 .\end{cases}\right.
$$

where $\mu_{n-}, \mu_{n+}, \mu_{p-}$, and $\mu_{p+}$ are constants.

\footnotetext{
${ }^{1}$ These functions are also sometimes termed the electrochemical potentials; this is confusing since the more standard definition of the electrochemical potentials includes an entropic contribution which is a function (typically a log) of the species concentration.
} 
2.2. Organic-organic interface. Two key features must be taken into consideration at the interface between two organic semiconductors. First there is a jump in electron affinity and the ionization potential, and in order to preserve equilibrium at the interface, this results in jumps in the electron and hole concentrations. Localized interface charged states have been postulated at the interface (e.g., [1]), but here we ignore such states and explicitly neglect the effect of any surface dipoles or charges so that the electric potential and displacement field are both continuous across the interface. Therefore we can write the jump conditions

$$
\begin{gathered}
{[\phi]_{x=0}, \quad\left[\varepsilon \frac{\partial \phi}{\partial x}\right]_{x=0}=0,} \\
{[\log n]_{x=0}=-\left[\frac{\mu_{n}}{k T}\right]_{x=0}, \quad[\log p]_{x=0}=\left[\frac{\mu_{p}}{k T}\right]_{x=0},}
\end{gathered}
$$

a result that has been previously noted in [5].

The second important feature of the organic-organic interface is that this is where recombination, thermal generation, and photo-generation occur in the device. (For brevity we shall henceforth refer to recombination and thermal generation as the recombination rate.) Since the ionization potential and electron affinity differ by a large amount the materials are nearly insulators, and there is negligible recombination of electrons and holes in the bulk of the device. Electrons from the acceptor side are able to recombine with holes from the donor side (at the interface) and vice versa. The resulting recombination flux $R_{b}$ results in jumps in the electron and hole current fluxes. This is central to the behavior of this type of solar cell - this mechanism allows the electron current in the acceptor to turn into a hole current in the donor. We expect the recombination rate to depend on the product of electron densities at $x=0_{-}$and the holes at $x=0_{+}$, namely, $\left(\left.n\right|_{x=0^{-}}\right)\left(\left.p\right|_{x=0^{+}}\right)$(or alternatively $\left(\left.n\right|_{x=0^{+}}\right)\left(\left.p\right|_{x=0^{-}}\right)$). A general expression could be derived, but because of the conditions in (2.11) the electron densities at $x=0_{-}$are directly proportional to those at $x=0_{+}$and similarly for holes. This allows us a certain freedom in choosing the form of the interface recombination rate in terms of the charge densities on either side of the interface. Since we expect that the most important role is played by majority carriers (electrons in the acceptor and holes in donor) we express the recombination flux in terms of those densities only. Hence we take

$$
\begin{aligned}
& {\left[\mathcal{F}_{n}\right]_{x=0}=-R_{b}\left(\left.n\right|_{x=0^{-}},\left.p\right|_{x=0^{+}}\right),} \\
& {\left[\mathcal{F}_{p}\right]_{x=0}=-R_{b}\left(\left.n\right|_{x=0^{-}},\left.p\right|_{x=0^{+}}\right) .}
\end{aligned}
$$

2.3. Existence of an equilibrium state and boundary conditions. Before considering what are sensible boundary conditions we consider the existence of an equilibrium state of (2.1)-(2.7). An equilibrium state is a steady state where both the electron and hole fluxes are simultaneously zero, that is, $\mathcal{F}_{n}^{\text {equil }} \equiv 0$ and $\mathcal{F}_{p}^{\text {equil }} \equiv 0$. Therefore from (2.4)-(2.5) with (2.11) it follows that $n$ and $p$ take the form

$$
n=A \exp \left(-\frac{1}{k T} \psi_{n}(x)\right), \quad p=B \exp \left(\frac{1}{k T} \psi_{p}(x)\right)
$$

for some constants $A$ and $B$. We note that this solution has a special property which we find by multiplying the equilibrium concentrations to give (noting that the potential terms cancel)

$$
n p=A B \exp \left(-\frac{1}{k T}\left(\mu_{n}(x)-\mu_{p}(x)\right)\right) .
$$


The right-hand side of (2.14) only depends on material properties, so we introduce the notation

$$
n p=N_{D}^{2} \exp \left(-\frac{1}{k T}\left(\mu_{n}(x)-\mu_{p}(x)\right)\right)
$$

where we write $A B=N_{D}^{2}$, and this relates to the density of states in the material.

The equilibrium condition in the dark occurs when no applied potential is present. Since different metals are typically used to contact the electron and donor materials, the potential difference across the device consists of both the applied potential $V$ and a built-in potential $V_{b i}$ which results from the difference of work functions of the two metallic contacts. (If the same metal is used for both contacts, then $V_{b i}=0$.) At equilibrium, when by definition $V=0$, the boundary conditions for the (total) potential is $\left.\phi\right|_{x=-L}=V_{b i} / 2$ and $\left.\phi\right|_{x=M}=-V_{b i} / 2$; then

$$
\begin{aligned}
& n=\left.A \exp \left(-\frac{1}{k T}\left(\mu_{n-}-q \frac{V_{b i}}{2}\right)\right)\right|_{x=-L}, \\
& p=\left.B \exp \left(\frac{1}{k T}\left(\mu_{p-}-q \frac{V_{b i}}{2}\right)\right)\right|_{x=-L}, \\
& n=\left.A \exp \left(-\frac{1}{k T}\left(\mu_{n+}+q \frac{V_{b i}}{2}\right)\right)\right|_{x=M} \\
& p=\left.B \exp \left(\frac{1}{k T}\left(\mu_{p+}+q \frac{V_{b i}}{2}\right)\right)\right|_{x=M}
\end{aligned}
$$

These equations show the role of the built-in voltage: it allows large concentrations of electrons (or holes) at the contacts. The diffusion arising from these large charge densities must be balanced by a drift term; therefore a built-in voltage allows an equilibrium state to exist with nonzero electric field.

The recombination and thermal generation rate. The need for an equilibrium also partly determines the functional form that the recombination at the interface must take. Since both electron and hole fluxes must be zero at equilibrium, the net recombination and thermal generation rate $R_{b}$ must also be zero in this case. It is natural to therefore write $R_{b}$ as a product with

$$
R_{b}=K\left(\left.n\right|_{x=0^{-}},\left.p\right|_{x=0^{+}}\right)\left(\left(\left.n\right|_{x=0^{-}}\right)\left(\left.p\right|_{x=0^{+}}\right)-N_{D}^{2} \exp \left(-\frac{\mu_{n-}-\mu_{p+}}{k T}\right)\right)
$$

and $K(n, p)>0$. Here the rate can be seen as composed of two parts: the bimolecular recombination of electrons and holes (proportional to $\left.\left.n\right|_{x=0^{-}} p\right|_{x=0^{+}}$) and a thermal generation proportional to the intrinsic charge density $\left(-N_{D}^{2} \exp \left(-\frac{\mu_{n-}-\mu_{p+}}{k T}\right)\right)$. Henceforth, for brevity, we refer to the net recombination and thermal generation rate as the recombination rate. Since other properties also depend on the difference of electron affinity in the acceptor and the ionization potential in the donor, we define this quantity as the pseudo band gap $E_{g}=\mu_{n-}-\mu_{p+}$. Notice that this is not a band gap in the conventional sense: usually a band gap is a property of a single material such as the difference between the electron affinity and the ionization potential, whereas in this case the pseudo band gap relates to the pair of materials. However, because it defines the temperature dependence of the thermal generation of charges, it plays a very similar role to the band gap in a traditional semiconductor. Writing the recombination at the interface in the form of (2.17) allows us considerable freedom in the choice 
of the function $K$. It could be a constant, giving us direct recombination, or it could be proportional to the inverse of the charge densities, giving a Shockley-Read-Hall (SRH) recombination. It could also depend on the electric field or on temperature. $\mathrm{SRH}$ is appropriate for recombination via a single trap, but for an organic device it may be more appropriate to modify this to account for a distribution of traps (see, for example, [13]). Henceforth we assume SRH recombination by writing

$$
R_{b}=K_{0}\left(\frac{\left(\left.n\right|_{x=0^{-}}\right)\left(\left.p\right|_{x=0^{+}}\right)-N_{D}^{2} \exp \left(-E_{g} /(k T)\right)}{1+\left.u_{1} n\right|_{x=0^{-}}+\left.u_{2} p\right|_{x=0^{+}}}\right)
$$

where $K_{0}, u_{1}$, and $u_{2}$ are appropriately chosen constants. This can be interpreted as recombination controlled by a single intermediate state at the interface. If different forms of the recombination were used, the form of some of the parameters in the asymptotic analysis which follows would change, but this would not change the general thrust of the model.

Boundary conditions. At the boundaries it is typically assumed that there are many surface states so that local equilibrium is always retained. This is obviously compatible with the need to have an equilibrium solution (2.16). The resulting boundary conditions at the contacts with the electrodes are

$$
\begin{gathered}
\left.n p\right|_{x=-L}=N_{D}^{2} \exp \left(-\frac{1}{k T}\left(\mu_{n-}-\mu_{p-}\right)\right), \\
\left.n p\right|_{x=M}=N_{D}^{2} \exp \left(-\frac{1}{k T}\left(\mu_{n+}-\mu_{p+}\right)\right) .
\end{gathered}
$$

The final boundary conditions come from imposing the potential at the contacts, the electron concentration on $x=-L$, and ensuring that the conditions (2.16) are satisfied; they take the form

$$
\begin{aligned}
& n=\tilde{n}_{-} \exp \left(-\frac{E_{g}}{2 k T}+\frac{q V_{b i}}{2 k T}\right) \quad \text { and } \quad \phi=\frac{V+V_{b i}}{2} \quad \text { on } \quad x=-L, \\
& p=\frac{N_{D}^{2}}{\tilde{n}_{-}} \exp \left(-\frac{E_{g}}{2 k T}+\frac{q V_{b i}}{2 k T}\right) \quad \text { and } \quad \phi=-\frac{V+V_{b i}}{2} \quad \text { on } \quad x=M .
\end{aligned}
$$

The condition on $p$ is equivalent to the assumption of Ohmic contacts (namely, that the Fermi level in the semiconductor is assumed equal to the work function of the metal) resulting in an electron and hole density which is independent of the applied potential. Without loss of generality the boundary conditions on the potential have been chosen to be antisymmetric in order to simplify later calculations.

2.4. Nondimensionalization. In order to analyze the problem we introduce dimensionless variables, indicated by the superscript ${ }^{*}$. This allows us to consider physically relevant ranges for the resulting dimensional parameters. For most of the variables the scalings are straightforward. We take

$$
\begin{aligned}
& x=L x^{*}, \quad t=\frac{L^{2}}{\bar{D}} t^{*}, \quad n=\Pi_{0} n^{*}, \quad p=\Pi_{0} p^{*}, \\
& (2.22) \psi_{p}=\frac{\mu_{p-}+\mu_{p+}}{2}+k T \psi_{p}^{*}, \quad \phi=\frac{k T}{q} \phi^{*}, \quad \varepsilon=\bar{\varepsilon} \varepsilon^{*}, \\
& \psi_{n}=\frac{\mu_{n-}+\mu_{n+}}{2}+k T \psi_{n}^{*}, \quad \mathcal{F}_{n}=\frac{\bar{D} \Pi_{0}}{L} \mathcal{F}_{n}^{*}, \quad \mathcal{F}_{p}=\frac{\bar{D} \Pi_{0}}{L} \mathcal{F}_{p}^{*}, \quad J=\frac{\bar{D} \Pi_{0} q}{L} J^{*},
\end{aligned}
$$


where the constant $\bar{\varepsilon}$ is a typical permittivity and $\bar{D}$ is a typical diffusivity, and we choose the typical electron-hole concentration, $\Pi_{0}$, to be given by

$$
\Pi_{0}=N_{D} \exp \left(-\frac{E_{g}}{2 k T}\right),
$$

which has a role similar to the intrinsic carrier concentration used in inorganic semiconductor theory.

On substituting the above into (2.1)-(2.7) and (2.18)-(2.21) we obtain the following dimensionless equations:

$$
\begin{aligned}
\frac{\partial n^{*}}{\partial t^{*}}+\frac{\partial \mathcal{F}_{n}^{*}}{\partial x^{*}} & =0 \\
\frac{\partial p^{*}}{\partial t^{*}}+\frac{\partial \mathcal{F}_{p}^{*}}{\partial x^{*}} & =0 \\
\frac{\partial}{\partial x^{*}}\left(\varepsilon^{*} \frac{\partial \phi^{*}}{\partial x^{*}}\right) & =\frac{1}{\lambda^{2}}\left(n^{*}-p^{*}\right), \\
\mathcal{F}_{n}^{*} & =-\kappa_{n} \exp \left(\nu\left|\frac{\partial \phi^{*}}{\partial x^{*}}\right|^{1 / 2}\right)\left(\frac{\partial n^{*}}{\partial x^{*}}-n^{*} \frac{\partial \phi^{*}}{\partial x^{*}}\right), \\
\mathcal{F}_{p}^{*} & =-\kappa_{p} \exp \left(\nu\left|\frac{\partial \phi^{*}}{\partial x^{*}}\right|^{1 / 2}\right)\left(\frac{\partial p^{*}}{\partial x^{*}}+p^{*} \frac{\partial \phi *}{\partial x^{*}}\right)
\end{aligned}
$$

with the jump conditions on the interface

$$
\left[\phi^{*}\right]_{x^{*}=0}=0, \quad\left[\varepsilon^{*} \frac{\partial \phi^{*}}{\partial x^{*}}\right]_{x^{*}=0}=0, \quad\left[\log n^{*}\right]_{x^{*}=0}=-H_{n}, \quad\left[\log p^{*}\right]_{x^{*}=0}=H_{p},
$$

$$
\left[\mathcal{F}_{n}^{*}\right]_{x^{*}=0}=\left[\mathcal{F}_{p}^{*}\right]_{x^{*}=0}=-2 \delta\left(\frac{\left.\left.n^{*}\right|_{x^{*}=0^{-}} p^{*}\right|_{x^{*}=0^{+}}-1}{\lambda^{2} \theta+\left.U n^{*}\right|_{x=0^{-}}+\left.(1-U) p^{*}\right|_{x=0^{+}}}\right),
$$

and the boundary conditions

$$
\begin{aligned}
\left.n^{*}\right|_{x^{*}=-1} & =\tilde{N}_{-} \exp \left(\frac{\Phi_{b i}}{2}\right), \\
\left.p^{*}\right|_{x^{*}=-1} & =\frac{1}{\tilde{N}_{-}} \exp \left(-\frac{\Phi_{b i}}{2}-H_{p}\right),\left.\quad \phi^{*}\right|_{x^{*}=-1}=\frac{\Phi+\Phi_{b i}}{2}, \\
\left.p^{*}\right|_{x^{*}=m} & =\frac{1}{\tilde{N}_{-}} \exp \left(\frac{\Phi_{b i}}{2}\right), \\
\left.n^{*}\right|_{x^{*}=m} & =\tilde{N}_{-} \exp \left(-\frac{\Phi_{b i}}{2}-H_{n}\right),\left.\quad \phi^{*}\right|_{x^{*}=m}=-\frac{\Phi+\Phi_{b i}}{2} .
\end{aligned}
$$

The total current density is

$$
J^{*}=\mathcal{F}_{p}^{*}-\mathcal{F}_{n}^{*},
$$

and the dimensionless energies of the LUMO and HOMO are

$$
\left.\left.\begin{array}{l}
\psi_{n}^{*}=-\frac{H_{n}}{2}-\phi^{*} \\
\psi_{p}^{*}=-\frac{H_{p}}{2}-\phi^{*}
\end{array}\right\} \text { in } x<0 \quad \text { and } \quad \begin{array}{l}
\psi_{n}^{*}=\frac{H_{n}}{2}-\phi^{*} \\
\psi_{p}^{*}=\frac{H_{p}}{2}-\phi^{*}
\end{array}\right\} \text { in } x>0 .
$$


The dimensionless parameters in the problem are defined by

$$
\begin{array}{ccc}
\kappa_{n}=\frac{D_{n 0}}{\bar{D}}, & \theta=\frac{1}{\lambda^{2} N_{D}\left(u_{1}+u_{2}\right)} \exp \left(\frac{-E_{g}}{2 k T}\right), & \lambda=\frac{1}{L}\left(\frac{\bar{\varepsilon} k T}{q^{2} \Pi_{0}}\right)^{1 / 2}, \\
\kappa_{p}=\frac{D_{p 0}}{\bar{D}}, & \tilde{N}_{-}=\frac{\tilde{n}_{-}}{N_{D}}, & \Phi=\frac{q}{k T} V, \\
\nu=\gamma\left(\frac{k T}{q L}\right)^{1 / 2}, & U=\frac{u_{1}}{u_{1}+u_{2}}, & \delta=\frac{L K_{0}}{2 \bar{D}\left(u_{1}+u_{2}\right)}, \\
m=\frac{M}{L}, & H_{n}=\frac{\mu_{n+}-\mu_{n-}}{k T}, & H_{p}=\frac{\mu_{p+}-\mu_{p-}}{k T}, \\
\Phi_{b i}=\frac{q}{k T} V_{b i} . &
\end{array}
$$

We use existing physical data to consider the size of the various parameters. A typical electron-hole concentration is, according to [1], $\Pi_{0}=O\left(10^{22}\right) \mathrm{m}^{-3}$, device width is about $L=O\left(10^{-7}\right) \mathrm{m}$, and the permittivity $\bar{\varepsilon}$ is similar to the permittivity of free space $\varepsilon_{0}=8.85 \times 10^{-12} \mathrm{~A} \mathrm{sV}^{-1} \mathrm{~m}^{-1}$. Buxton and Clarke [3] take $\gamma=5 \times$ $10^{-4} \mathrm{~m}^{1 / 2} \mathrm{~V}^{-1 / 2}$. The charge on an electron is $q=1.6 \times 10^{-19} \mathrm{C}$. Using these numbers we estimate the parameters in the model as

$$
\lambda \approx O(1), \quad \gamma \approx O(1), \quad H_{n} \gtrsim 50, \quad H_{p} \gtrsim 50
$$

and will therefore exploit the largeness of $H_{n}$ and $H_{p}$ in our analysis. Other parameters in the problem are less well documented, and part of the motivation for the analysis done here is to identify how the general behavior depends on these values. For most parameters we shall assume that they do not take extreme values, however, we find that the dependency of the voltage on the current is critically altered by the value of $\delta$ (the ratio of the recombination rate to the diffusion rate). Unless $\delta$ is very small the voltage-current characteristic does not have the behavior observed in real devices (e.g., [19]). We therefore make the assumption that $\delta$ is very small in our analysis and indeed we find that the size of $\delta$ is crucial in determining the electrical behavior of the device.

Note that from here on we drop the superscript * notation from the dimensionless variables.

2.5. Simplifications: Large jumps in electron affinity, no Poole-Frenkel behavior, and an antisymmetric device. Using materials that are strong electron donors and electron acceptors (i.e., $H_{n} \gg 1$ and $H_{p} \gg 1$ ) means that the concentration of holes in the acceptor material and of electrons in the donor material are extremely small. In a silicon device these minority carriers are responsible for carrying much of the current, whereas in an organic device their role is insignificant. This is because it is possible for the majority carriers in the donor (holes) to recombine with the majority carriers in the acceptor (electrons) at the interface between the two organic semiconductors. This corresponds to the major simplification that the electron concentration in the donor material and the hole concentration in the acceptor material of an organic diode are both zero,

$$
p \equiv 0 \quad \text { in } \quad x<0 \text { and } \quad n \equiv 0 \quad \text { in } \quad x>0,
$$

which is justified by the extremely small sizes of $\exp \left(-H_{p}\right)$ and $\exp \left(-H_{n}\right)$. We also make three other simplifications: that the Poole-Frenkel behavior is insignificant; 
that the device is at steady state; and that the device is perfectly antisymmetric, corresponding to

$$
\nu=0, \quad \varepsilon_{-}=\varepsilon_{+}, \quad \kappa_{p}=\kappa_{n}, \quad \tilde{N}_{-}=1, \quad m=1,
$$

and by suitable choice of $\bar{\varepsilon}$ and $\bar{D}$ that specifically

$$
\varepsilon_{-}=\varepsilon_{+}=1, \quad \kappa_{p}=\kappa_{n}=1 .
$$

By assuming that the device is antisymmetric we can deduce that $n(-x)=p(x)$ (for $0<x<1$ ) and that the electric potential distribution is antisymmetric $\phi(-x)=$ $-\phi(x)$. These simplifications allow a straightforward analysis of the problem, avoiding much of the algebraic complexity, and reveal the general structure of the solution for practical applications. In particular, the neglect of the Poole-Frenkel effect simplifies the analysis without making major qualitative changes to the device behavior. We discuss its inclusion in the conclusions.

After making these simplifications and considering only half the antisymmetric device, the model takes the form

$$
\begin{gathered}
\left.\begin{array}{c}
\frac{\partial p}{\partial x}+p \frac{\partial \phi}{\partial x}=-J \\
\frac{\partial^{2} \phi}{\partial x^{2}}=-\frac{p}{\lambda^{2}}
\end{array}\right\} \text { in } \quad 0<x<1, \\
J=-\frac{2 \delta\left(\left.p^{2}\right|_{x=0}-1\right)}{\left(\lambda^{2} \theta+\left.p\right|_{x=0}\right)}, \\
\left.p\right|_{x=1}=\exp \left(\frac{\Phi_{b i}}{2}\right),\left.\quad \phi\right|_{x=1}=-\frac{\Phi+\Phi_{b i}}{2},\left.\quad \phi\right|_{x=0}=0 .
\end{gathered}
$$

3. Asymptotic solution of simplified model. We now attempt to find approximate solutions to the simplified model. In doing this we shall exploit the fact that $\delta$ is an extremely small number in practical devices. We justify this assumption by noting that in efficient devices the current varies by many orders of magnitude as the potential across the device is varied from hard reverse bias to hard forward bias. (In, for example, the devices fabricated by Potscavage, Yoo, and Kippelen [19] there is a variation in the magnitude of the current by a factor of about $10^{6}$ from hard reverse bias to hard forward bias.) As we shall demonstrate it is possible to achieve such large variations in the current only if recombination at the interface between the two semiconductors is very difficult (i.e., $\delta \ll 1$ ). If the recombination is easy the device acts predominantly as an Ohmic resitor. All other parameters are taken to be order one except for the voltage $\Phi$ and the current $J$ which we vary in order to find the relationship between $\Phi$ and $J$. In fact we will need to consider different regimes based on the sizes of these two parameters, relative to $\delta$. We remark that the dimensionless built-in potential $\Phi_{b i}=q V_{b i} /(k T)$ is typically large in practice but that in comparison to $\log (1 / \delta)$ we still expect it to be small. Furthermore, we assert that under these circumstances investigating the distinguished limit $\Phi_{b i}=O(1)$ still yields valid results.

We start by defining the new quantities

$$
N_{a}=\frac{1}{\lambda^{2}}, \quad \hat{J}=\frac{J}{2 \lambda^{2}}
$$


and substituting for $p$ (in terms of $\phi$ ) in (2.37)-(2.39); this enables us to rewrite these equations in the form

$$
\begin{gathered}
\frac{\partial^{3} \phi}{\partial x^{3}}+\frac{\partial \phi}{\partial x} \frac{\partial^{2} \phi}{\partial x^{2}}=2 \hat{J} \quad \text { in } \quad 0<x<1, \\
\phi=0 \quad \text { and } \quad \hat{J}=-\delta \frac{\phi_{x x}^{2}-N_{a}^{2}}{\theta-\phi_{x x}} \quad \text { on } \quad x=0, \\
\phi=-\frac{\Phi+\Phi_{b i}}{2} \quad \text { and } \quad \frac{\partial^{2} \phi}{\partial x^{2}}=-\exp \left(\frac{\Phi_{b i}}{2}\right) N_{a} \quad \text { on } \quad x=1 .
\end{gathered}
$$

We now proceed to solve this ODE problem in the limits of small and large currents to obtain the relevant current-voltage characteristics.

3.1. Small current limit. When the current is small, the system lies close to the equilibrium solution in which drift and diffusion components of the current density are large compared to the total current density and are almost in balance. Physically this is a regime in which the current in the bulk of the semiconductors is small and is determined by the rate of recombination at the interface. Formally we investigate the distinguished asymptotic limit $\hat{J}=O(\delta)$ and $\Phi+\Phi_{b i}=O(1)$ but note that the approximate solution we derive is asymptotically valid provided $\hat{J} \ll 1$; this is equivalent to requiring $-\Phi \ll \log (1 / \delta)$. Hence we look for an asymptotic solution to $(3.2)-(3.4)$ of the form

$$
\phi=\phi_{0}+\delta \phi_{1}+\cdots, \quad \hat{J}=\delta \hat{J}_{1}+\cdots .
$$

Substituting this ansatz into (3.2)-(3.4) yields the following problem at leading order:

$$
\begin{gathered}
\frac{\partial^{3} \phi_{0}}{\partial x^{3}}+\frac{\partial \phi_{0}}{\partial x} \frac{\partial^{2} \phi_{0}}{\partial x^{2}}=0 \quad \text { in } \quad x>0, \\
\phi_{0}=0 \quad \text { and } \quad \hat{J}_{1}=-\frac{\phi_{0 x x}^{2}-N_{a}^{2}}{\theta-\phi_{0 x x}} \quad \text { on } \quad x=0, \\
\quad \text { and } \quad \frac{\partial^{2} \phi_{0}}{\partial x^{2}}=-N_{a} \exp \left(\frac{\Phi_{b i}}{2}\right) \quad \text { on } \quad x=1 .
\end{gathered}
$$

Integrating (3.5) once and applying the boundary conditions (3.7) gives the relation

$$
\frac{\partial^{2} \phi_{0}}{\partial x^{2}}=-N_{a} \exp \left(-\frac{\Phi}{2}\right) \exp \left(-\phi_{0}\right) .
$$

This is all we need in order to derive the current-voltage curve since it allows us to evaluate $\left.\phi_{0, x x}\right|_{x=0}$ using the fact that $\left.\phi_{0}\right|_{x=0}=0$ and hence to find $\hat{J}_{1}$ from (3.6b). This yields

$$
\hat{J}_{1}=\frac{2 N_{a}^{2} \sinh (\Phi / 2)}{N_{a}+\theta \exp (\Phi / 2)} .
$$

Thus this part of the current-voltage curve is given, on substituting for $\hat{J}$ and $N_{a}$ from (3.1), by

$$
J \sim 4 \delta \frac{\sinh (\Phi / 2)}{1+\lambda^{2} \theta \exp (\Phi / 2)} .
$$


The solution for $\phi_{0}$. Solving for $\phi_{0}$ from (3.8), together with the boundary conditions (3.6a) and (3.7a), yields a solution that takes either the form

$$
\phi_{0}=-2 \log \left(\frac{\sinh (p)}{\sinh (p \pm A x))}\right), \quad \text { where } A \text { and } p \text { satisfy }
$$

$$
\frac{\sinh ^{2} p}{\sinh ^{2}(p \pm A)}=\exp \left(\frac{\Phi+\Phi_{b i}}{2}\right) \quad \text { and } \quad \frac{2 A^{2}}{\sinh ^{2}(p \pm A)}=N_{a} \exp \left(\frac{\Phi_{b i}}{2}\right)
$$

or the form

$$
\begin{gathered}
\phi_{0}=-2 \log \left(\frac{\sin q}{\sin (q \pm B x)}\right), \quad \text { where } B \text { and } q \text { satisfy } \\
\frac{\sin ^{2} q}{\sin ^{2}(q \pm B)}=\exp \left(\frac{\Phi+\Phi_{b i}}{2}\right) \text { and } \frac{2 B^{2}}{\sin ^{2}(q \pm B)}=N_{a} \exp \left(\frac{\Phi_{b i}}{2}\right) .
\end{gathered}
$$

Thus if $N_{a} \exp \left(\frac{\Phi_{b i}}{2}\right)<2$, the appropriate form of the solution is (3.11)-(3.12), while if $N_{a} \exp \left(\frac{\Phi_{b i}}{2}\right)>2$, the appropriate form of the solution is (3.13)-(3.14).

Reverse bias. In hard reverse bias when the applied voltage is much greater than $\Phi_{b i}(3.10)$ reduces to

$$
\hat{J}_{1} \sim \frac{N_{a}^{2}}{\theta} \quad \text { as } \quad \Phi \rightarrow \infty
$$

This corresponds to the reverse saturation current in a traditional Shottky diode. It is also possible to look at the potential distribution in these conditions. This is done by solving (3.12) and approximating the hyperbolic arc cosecant function by a logarithm. The potential can then be written as

$$
\phi_{0} \sim-\frac{\Phi}{2} x .
$$

A physical picture is easy to glean: in reverse bias some charges are generated thermally at the interface and are being removed by a constant electric field. Since in our model the thermally generated current is voltage independent, the reverse current saturates. Figure 3.1(II) shows this approximate solution and the corresponding numerical solution for the dimensionless energy levels of the LUMO and HOMO $\left(\psi_{n}\right.$ and $\psi_{p}$, respectively) as defined in (2.32).

Forward bias. In forward bias, the potential is large and negative, and in this case (3.10) can be approximated by an exponential:

$$
\hat{J}_{1} \sim-N_{a} \exp \left(-\frac{\Phi}{2}\right) \quad \text { as } \Phi \rightarrow-\infty .
$$

This situation is very similar to the standard solution to the Shockley equation where the forward bias current is written as $J \propto \exp (-\Phi / n)$, where $n$ is the so-called ideality factor. The ideality factor we obtain here is therefore equal to 2 . This is because in the limit of large charge density and in an antisymmetric configuration, the SRH recombination term varies as the charge density. Since the charge density at the interface is proportional to $\exp (\Phi / 2)$, the ideality factor is 2 . If we had used bare recombination, where the recombination flux is proportional to charge density squared, we would have obtained an ideality factor of 1 . 


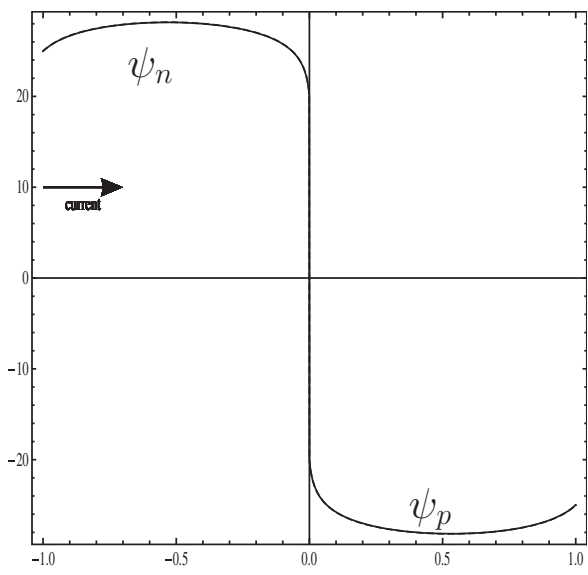

(I)

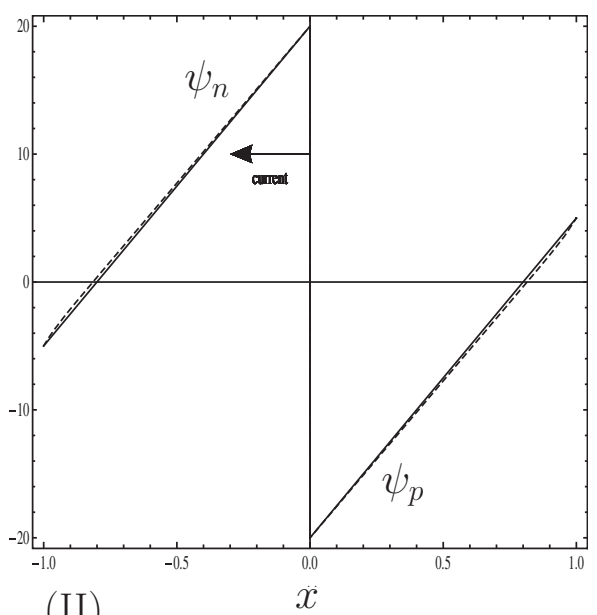

(II)

Fig. 3.1. Diagrams showing the dimensionless energies of the $L U M O, \psi_{n}$, in $x<0$ and the $H O M O, \psi_{p}$, in $x>0$. Both diagrams assume a pseudo band gap $E_{g}$ of $40(1 \mathrm{eV})$ and $\Phi_{b i}=12$ with $\lambda=1$. However, in (I) $\Phi=-22$ corresponding to a device in forward bias, while in (II) $\Phi=38$ corresponding to a device in reverse bias. Shown are solutions calculated directly from the numerical solution to (3.12) (dotted lines) and ones derived from the approximate solutions (3.19), in forward bias, and (3.16), in reverse bias (solid lines).

By assuming that both $-\Phi \gg 1$ it is again possible to obtain approximate expressions for the potential distribution both in an exponentially narrow layer in the immediate vicinity of the junction (i.e., for $x=O(\exp (-|\Phi| / 4)))$ and away from it (i.e., for $x=O(1)){ }^{2}$ Rather than write both these expressions we write a uniformly valid asymptotic expression for the potential throughout $0 \leq x \leq 1$,

$$
\begin{gathered}
\phi_{0} \sim-2 \log \left(\frac{\pi \xi_{2} e^{-|\Phi| / 4}}{\sin \left(\pi\left(x+\xi_{2} e^{-|\Phi| / 4}\right)\right)}\right), \\
\text { where } \xi_{2}=\sqrt{\frac{2}{N_{a}}} \text { and } \xi_{2} \ll 1 .
\end{gathered}
$$

This form of the solution is appropriate for studying both the potential distribution at equilibrium and in forward bias. An example showing the dimensionless energy levels of LUMO and HOMO in forward bias (calculated from (3.19) and (2.32)) is shown in Figure 3.1(I). Here, as the bias is increased, the levels bend further and further at the interface, increasing the charge density. The increase in charge density drives a greater current through the device. At forward bias electrons are injected in the donor, holes are injected in the acceptor, and they annihilate at the interface.

3.1.1. The large current large potential asymptotics $-\hat{J}=O(\log (1 / \delta))$, $-\Phi=O(\log (1 / \delta))$. As the voltage becomes larger in magnitude (in forward bias), the charge density at the interface increases exponentially as does the current. This increase in current can only continue in quasi-static equilibrium so long as the increase in drift current toward the interface can be matched by a similar increase in diffusion

\footnotetext{
${ }^{2}$ The asymptotic expansion remains valid provided $\left|\delta \hat{J}_{1}\right| \ll 1$, which is equivalent to the condition $|\Phi| \ll 2 \log \left(N_{a} / \delta\right)$.
} 
away from the interface. Such a diffusion relies on draining the bulk of the semiconductor away from the interface of charges, and this cannot be sustained indefinitely. For sufficiently large voltage the current is controlled by the transport in the bulk of the device.

Here we write $\hat{J}=\log (1 / \delta) \hat{J}_{*}$, where $\hat{J}_{*}<0$ and $-\hat{J}_{*}=O(1)$. In terms of this new variable the steady state equations are

(3.21) $\phi=-\frac{\Phi+\Phi_{b i}}{2}$

$$
\begin{aligned}
\frac{\partial^{3} \phi}{\partial x^{3}}+\frac{\partial \phi}{\partial x} \frac{\partial^{2} \phi}{\partial x^{2}}=-2\left|\hat{J}_{*}\right| \log \left(\frac{1}{\delta}\right) & \text { in } \quad x>0, \\
\phi=0 \quad \text { and } \quad\left|\hat{J}_{*}\right|=\frac{\delta}{\log (1 / \delta)} \frac{\phi_{x x}^{2}-N_{a}^{2}}{\theta-\phi_{x x}} & \text { on } \quad x=0, \\
\text { and } \quad \frac{\partial^{2} \phi}{\partial x^{2}}=-N_{a} \exp \left(\Phi_{b i} / 2\right) & \text { on } \quad x=1 .
\end{aligned}
$$

In order to study this problem we need to investigate the boundary layer about $x=0$ whose width is found by balancing the right-hand side of $(3.20 \mathrm{~b})$ with the modulus of the current density $\left|\hat{J}_{*}\right|$.

Inner region (II). The role of this boundary layer region is to match the charge density to the recombination condition. This is a region in which a quasi equilibrium holds with a high concentration of charge and is reminiscent of an inversion layer. Immediately adjacent to the semiconductor interface on $x=0$ we introduce the scaled variable $\eta$ which is related to $x$ via

$$
x=\frac{\delta^{1 / 2}}{(\log (1 / \delta))^{1 / 2}} \eta .
$$

In terms of this new variable the equations and boundary conditions (3.19)-(3.20) become

$$
\begin{array}{r}
\frac{\partial^{3} \phi}{\partial \eta^{3}}+\frac{\partial \phi}{\partial \eta} \frac{\partial^{2} \phi}{\partial \eta^{2}}=O\left(\delta^{3 / 2} \log \left(\frac{1}{\delta}\right)^{-1 / 2}\right) \quad \text { in } \quad \eta>0, \\
\phi=0 \quad \text { and } \quad\left|\hat{J}_{*}\right|=-\frac{\phi_{\eta \eta}^{2}-\delta^{2}(\log (1 / \delta))^{-2} N_{a}^{2}}{\phi_{\eta \eta}-\delta(\log (1 / \delta))^{-1} \theta} \quad \text { on } \quad \eta=0 .
\end{array}
$$

Expanding $\phi$ as

$$
\phi^{(I I)}=\phi_{2}^{(I I)}+\cdots
$$

and substituting into (3.22)-(3.23) yields the leading order problem

$$
\begin{array}{r}
\frac{\partial^{3} \phi_{2}^{(I I)}}{\partial \eta^{3}}+\frac{\partial \phi_{2}^{(I I)}}{\partial \eta} \frac{\partial^{2} \phi_{2}^{(I I)}}{\partial \eta^{2}}=0, \\
\phi_{2}^{(I I)}=0 \quad \text { and } \quad \frac{\partial^{2} \phi_{2}^{(I I)}}{\partial \eta^{2}}=-\left|\hat{J}_{*}\right| \quad \text { on } \quad \eta=0
\end{array}
$$

with solution

$$
\phi_{2}^{(I I)}=2 \log \left(1+\left|\frac{\hat{J}_{*}}{2}\right|^{1 / 2} \eta\right) .
$$

We now require matching this to an outer region on the $O(1)$ lengthscale. However, in order to do this, we need to introduce an intermediate inner region and match across this region from the inner to the outer. In this intermediate inner region (as in the inner region) a quasi equilibrium exists. 
Intermediate inner region (I). Here we rescale about the interface on $x=0$ by introducing the new variable $\xi$, defined by

$$
x=\frac{\xi}{\log (1 / \delta)}, \quad\left(\xi=\delta^{1 / 2}(\log (1 / \delta))^{1 / 2} \eta\right)
$$

in terms of which (3.19) can be rewritten as

$$
\frac{\partial^{3} \phi}{\partial \xi^{3}}+\frac{\partial \phi}{\partial \xi} \frac{\partial^{2} \phi}{\partial \xi^{2}}=-\frac{2\left|\hat{J}_{*}\right|}{\log \left(\frac{1}{\delta}\right)^{2}} .
$$

Matching to the leading order solution in the inner region we see that the expansion for $\phi$ proceeds, in powers of $\delta$, as follows:

$$
\phi^{(I)}=\log \left(\frac{1}{\delta}\right)-\log \left(\log \left(\frac{1}{\delta}\right)\right)+\phi_{2}^{(I)}+\cdots .
$$
$\phi_{2}^{(I)}$ :

Proceeding to $O(1)$ in the expansion of (3.25) gives the following equation for

$$
\frac{\partial^{3} \phi_{2}^{(I)}}{\partial \xi^{3}}+\frac{\partial^{2} \phi_{2}^{(I)}}{\partial \xi^{2}} \frac{\partial \phi_{2}^{(I)}}{\partial \xi}=0
$$

with solution

$$
\phi_{2}^{(I)}=C+2 \log (\sinh (M \xi)),
$$

where the constants $C$ and $M$ are determined by the matching conditions. Matching to the leading order inner solution implies

$$
\phi_{2}^{(I)} \sim 2 \log \left(\left|\frac{\hat{J}_{*}}{2}\right|^{1 / 2} \xi\right) \quad \text { as } \quad \xi \rightarrow 0
$$

and thus implies that

$$
\phi_{2}^{(I)}=\log \left|\frac{\hat{J}_{*}}{2}\right|-2 \log M+2 \log (\sinh (M \xi)),
$$

where the remaining constant $M$ is determined by matching to the outer region.

The outer region $(o)$. Finally we get to the outer region in which we solve (3.19) with boundary condition (3.21) (where $x=O(1)$ ). This is the region where drift dominates: the semiconductor has a uniform charge density and a constant electric field dictated by the current. By matching to the solution in the intermediate inner region it becomes apparent that the asymptotic expansion of the solution in the outer region takes the form

$$
\begin{aligned}
\phi^{(o)} & =\log \left(\frac{1}{\delta}\right) \phi_{0}^{(o)}+\log \left(\log \left(\frac{1}{\delta}\right)\right) \phi_{1}^{(o)}+\phi_{2}^{(o)}+\cdots, \\
\Phi+\Phi_{b i} & =\log \left(\frac{1}{\delta}\right) \Xi_{0}+\log \left(\log \left(\frac{1}{\delta}\right)\right) \Xi_{1}+\Xi_{2}+\cdots .
\end{aligned}
$$


Substituting into (3.19) leads to the leading order equation $\phi_{0}^{(o)}{ }_{x x}=0$ with solution

$$
\phi_{0}^{(o)}=A x+B,
$$

where $A$ and $B$ are constants. Proceeding to $O(\log (1 / \delta))$ in (3.19) and (3.21b) gives

$$
\phi_{2}^{(o)}{ }_{x x}=-\frac{2\left|\hat{J}_{*}\right|}{A} \quad \text { and }\left.\quad \phi_{2}^{(o)}{ }_{x x}\right|_{x=1}=-N_{a} \exp \left(\Phi_{b i} / 2\right)
$$

from which we conclude that $A=2\left|\hat{J}_{*}\right| /\left(N_{a} \exp \left(\Phi_{b i} / 2\right)\right)$ so that

$$
\phi_{0}^{(o)}=\frac{2\left|\hat{J}_{*}\right|}{N_{a} \exp \left(\Phi_{b i} / 2\right)} x+B .
$$

Proceeding to $O(\log (1 / \delta) \log (\log (1 / \delta)))$ and to $O(\log (\log (1 / \delta)))$ leads to the result that

$$
\phi_{1}^{(o)}=K, \quad \Xi_{1}=-2 K,
$$

where $K$ is a constant.

Matching and the large current current-voltage curve. Matching between the solution in the intermediate region, (3.25), and the solution in the outer region, (3.27)(3.28), at the two lowest orders asserts that $B=1$ and $K=-1$. Hence, the two leading order terms in the outer region are uniquely determined as

$$
\phi_{0}^{(o)}=\frac{2\left|\hat{J}_{*}\right|}{N_{a} \exp \left(\Phi_{b i} / 2\right)} x+1, \quad \phi_{1}^{(o)}=-1 .
$$

Applying the boundary condition (3.21a) to this solution allows us to determine the two leading terms in the potential drop across the cell as

$$
\Xi_{0}=-\frac{4\left|\hat{J}_{*}\right|}{N_{a} \exp \left(\Phi_{b i} / 2\right)}-2 \text { and } \Xi_{1}=2
$$

Thus this part of the current-voltage curve is given, on substituting for $\hat{J}$ and $N_{a}$ from (3.1), by

$$
J \sim \frac{1}{2}\left(\left(\Phi+\Phi_{b i}\right)+2 \log (1 / \delta)-2 \log \left(\log \left(\frac{1}{\delta}\right)\right)\right) \exp \left(\Phi_{b i} / 2\right) .
$$

Notably $J$ varies linearly with $\Phi$, the result of Ohmic dissipation in the semiconductor bulk, which becomes significant for sufficiently large forwarded biased currents.

Matching the intermediate inner to the outer to find $M$. As $\xi \rightarrow \infty$ the solution (3.26) for $\phi_{2}^{(I)}$ (the second order intermediate inner) has the far field behavior

$$
\phi_{2}^{(I)} \sim 2 M \xi \quad \text { as } \quad \xi \rightarrow \infty .
$$

This term matches onto $x$-dependence of the leading order outer solution as $x \rightarrow 0$. Looking at (3.29) we can thus identify

$$
M=\frac{\left|\hat{J}_{*}\right|}{N_{a} \exp \left(\Phi_{b i} / 2\right)}
$$

so that $\phi_{2}^{(I)}$ is uniquely determined as

$$
\phi_{2}^{(I)}=\log \left|\frac{\hat{J}_{*}}{2}\right|-2 \log \left(\frac{\left|\hat{J}_{*}\right|}{N_{a} \exp \left(\Phi_{b i} / 2\right)}\right)+2 \log \left(\sinh \left(\frac{\left|\hat{J}_{*}\right|}{N_{a} \exp \left(\Phi_{b i} / 2\right)} \xi\right)\right) .
$$


3.1.2. The intermediate current asymptotics: $\hat{J}<0$ with $\hat{J}=O(1)$, $\Phi+\Phi_{b i}=-2 \log (\mathbf{1} / \delta)+\boldsymbol{O}(\mathbf{1})$. In order to complete the asymptotic description of the current-voltage curve we consider the intermediate current asymptotics. Essentially this is the regime in which the potential is too small for drift to deplete the charges in the bulk of the semiconductor, yet too large for quasi-static equilibrium to dominate the current-voltage characteristics. Here it is possible to find analytic solutions to differential equations for the drift diffusion process, but the transcendental equations that result from fitting this analytic solution to the boundary data have no explicit analytic solution.

Outer solution (o). Here we look for an asymptotic solution to (3.2) and (3.4) of the form

$$
\begin{aligned}
\phi^{(o)} & =\log \left(\frac{1}{\delta}\right) \phi_{0}^{(o)}+\phi_{1}^{(o)}+\cdots, \\
\Phi+\Phi_{b i} & =\log \left(\frac{1}{\delta}\right) \Xi_{0}+\Xi_{1}+\cdots .
\end{aligned}
$$

To leading order we find

$$
\phi_{0}^{(o)}=\text { constant }=-\frac{\Xi_{0}}{2},
$$

while at the next order we retrieve the following problem for $\phi_{1}^{(i)}$ :

$$
\begin{aligned}
\frac{\partial^{3} \phi_{1}^{(o)}}{\partial x^{3}}+\frac{\partial \phi_{1}^{(o)}}{\partial x} \frac{\partial^{2} \phi_{1}^{(o)}}{\partial x^{2}} & =-2|\hat{J}|, \\
\left.\frac{\partial^{2} \phi_{1}^{(o)}}{\partial x^{2}}\right|_{x=1} & =-N_{a} \exp \left(\frac{\Phi_{b i}}{2}\right),\left.\quad \phi_{1}^{(o)}\right|_{x=1}=-\frac{\Xi_{1}}{2} .
\end{aligned}
$$

In order to couple this solution to the boundary conditions on $x=0$ we need to introduce an inner region.

Inner region $(i)$. The inner variable $\nu$ is defined by

$$
x=\delta^{1 / 2} \nu
$$

such that (3.2)-(3.3) now read

$$
\begin{aligned}
& \quad \frac{\partial^{3} \phi}{\partial \nu^{3}}+\frac{\partial \phi}{\partial \nu} \frac{\partial^{2} \phi}{\partial \nu^{2}}=-2 \delta^{3 / 2}|\hat{J}| \text { in } \quad x>0, \\
& \phi=0 \quad \text { and } \quad|\hat{J}|=\frac{\phi_{\nu \nu}^{2}-\delta^{2} N_{a}^{2}}{\phi_{\nu \nu}-\delta \theta} \quad \text { on } \quad \nu=0 .
\end{aligned}
$$

We expand $\phi$ in the inner as follows:

$$
\phi^{(i)}=\phi_{1}^{(i)}+\cdots .
$$

At leading order $\phi_{1}^{(i)}$ satisfies the problem

$$
\frac{\partial^{3} \phi_{1}^{(i)}}{\partial \nu^{3}}+\frac{\partial \phi_{1}^{(i)}}{\partial \nu} \frac{\partial^{2} \phi_{1}^{(i)}}{\partial \nu^{2}}=0, \quad|\hat{J}|=-\left.\frac{\partial^{2} \phi_{1}^{(i)}}{\partial \nu^{2}}\right|_{\nu=0},\left.\quad \phi_{1}^{(i)}\right|_{\nu=0}=0
$$

with solution

$$
\phi_{1}^{(i)}=2 \log \left(1+\left|\frac{\hat{J}}{2}\right|^{1 / 2} \nu\right)
$$


Matching. Taking the large $\nu$ limit in (3.36) gives

$$
\phi_{1}^{(i)} \sim 2 \log \nu+\log \left|\frac{\hat{J}}{2}\right|+O\left(\frac{1}{\nu}\right) .
$$

Writing this in terms of the outer variable $\nu=x / \delta^{1 / 2}$ gives

$$
\phi_{1}^{(i)} \sim \log \left(\frac{1}{\delta}\right)+2 \log x+\log \left|\frac{\hat{J}}{2}\right|+O\left(\delta^{1 / 2}\right) .
$$

Matching to the outer problem at leading order gives

$$
\phi_{0}^{(o)}=1, \quad \Xi_{0}=-2 .
$$

At next order the matching condition (3.37) provides conditions that close (3.33), the outer problem for $\phi_{1}^{(o)}$

$$
\phi_{1}^{(o)} \sim 2 \log x+\log \left|\frac{\hat{J}}{2}\right| \quad \text { as } \quad x \rightarrow 0 .
$$

The full outer problem. This is found by combining the above matching condition with (3.33),

$$
\begin{gathered}
\frac{\partial^{3} \phi_{1}^{(o)}}{\partial x^{3}}+\frac{\partial \phi_{1}^{(o)}}{\partial x} \frac{\partial^{2} \phi_{1}^{(o)}}{\partial x^{2}}=-2|\hat{J}|, \\
\left.\frac{\partial^{2} \phi_{1}^{(o)}}{\partial x^{2}}\right|_{x=1}=-N_{a} \exp \left(\frac{\Phi_{b i}}{2}\right), \\
\phi_{1}^{(o)} \sim 2 \log x+\log \left|\frac{\hat{J}}{2}\right| \quad \text { as } \quad x \rightarrow 0
\end{gathered}
$$

with the correction to the potential being given by the solution to this problem via (3.33c). The exact solution to (3.39), which was independently derived in [2], is

$$
\phi_{1}^{(o)}=2 \log \left|\beta \operatorname{Ai}\left(|\hat{J}|^{1 / 3}\left(x_{1}-x\right)\right)+\gamma \operatorname{Bi}\left(|\hat{J}|^{1 / 3}\left(x_{1}-x\right)\right)\right|,
$$

where $x_{1}, \beta$, and $\gamma$ are arbitrary constants and $\operatorname{Ai}(\cdot)$ and $\operatorname{Bi}(\cdot)$ are Airy functions of the first and second kinds, respectively. Requiring that the solution satisfies the matching condition (3.41) determines $\gamma$ and $\beta$ so that the solution can be written in the form

$$
\begin{aligned}
\phi_{1}^{(o)}= & \log \left|\frac{\hat{J}}{2}\right| \\
& +2 \log \left|\frac{\operatorname{Bi}\left(|\hat{J}|^{1 / 3} x_{1}\right) \operatorname{Ai}\left(|\hat{J}|^{1 / 3}\left(x_{1}-x\right)\right)-\operatorname{Ai}\left(|\hat{J}|^{1 / 3} x_{1}\right) \operatorname{Bi}\left(|\hat{J}|^{1 / 3}\left(x_{1}-x\right)\right)}{|\hat{J}|^{1 / 3}\left(\operatorname{Ai}\left(|\hat{J}|^{1 / 3} x_{1}\right) \operatorname{Bi}^{\prime}\left(|\hat{J}|^{1 / 3} x_{1}\right)-\operatorname{Bi}\left(|\hat{J}|^{1 / 3} x_{1}\right) \operatorname{Ai}^{\prime}\left(|\hat{J}|^{1 / 3} x_{1}\right)\right)}\right| .
\end{aligned}
$$

$\operatorname{Ai}^{\prime}(\cdot)$ and $\operatorname{Bi}^{\prime}(\cdot)$ are the derivatives of the Airy functions of the first and second kinds, respectively. The final condition (3.40) leads to a rather complicated transcendental 

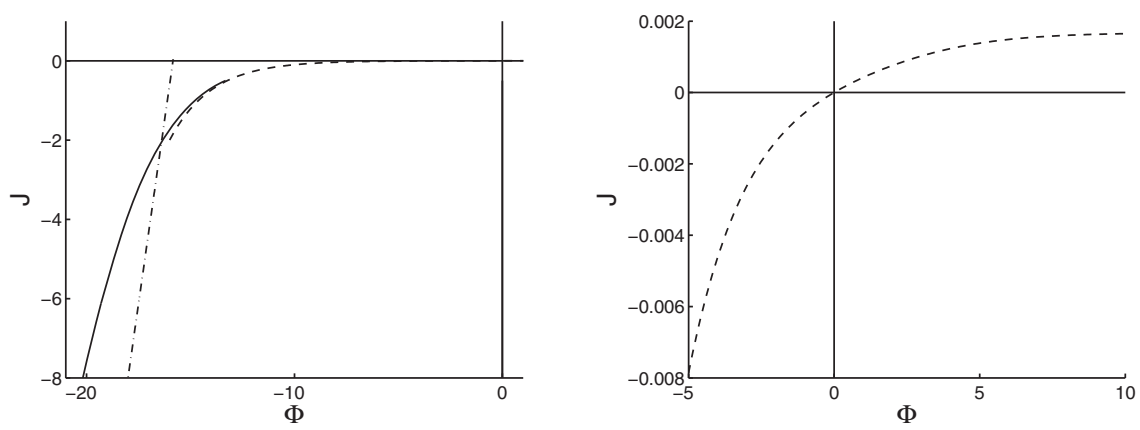

FIG. 3.2. Typical current-voltage curves derived from the low-current asymptotics (3.10)shown by dashed curve - from the intermediate asymptotics (3.42) - shown by a solid curve-and from the high-current asymptotics (3.31) - shown by a dot-dashed curve. Here $\Phi_{b i}=4, \delta=e^{-8}$, $\lambda=1$, and $\theta=0.4$. The left panel shows the low-, intermediate-, and high-current current-voltage curves. The right panel shows only the low-current approximation.

equation which can be solved to find the final parameter $x_{1}$. The correction to the potential is given by $(3.33 \mathrm{c})$ so that in this case

$$
\begin{aligned}
\Xi_{1}= & -2 \log \left|\frac{\hat{J}}{2}\right| \\
& -4 \log \left|\frac{\operatorname{Bi}\left(|\hat{J}|^{1 / 3} x_{1}\right) \operatorname{Ai}\left(|\hat{J}|^{1 / 3}\left(x_{1}-1\right)\right)-\operatorname{Ai}\left(|\hat{J}|^{1 / 3} x_{1}\right) \operatorname{Bi}\left(|\hat{J}|^{1 / 3}\left(x_{1}-1\right)\right)}{|\hat{J}|^{1 / 3}\left(\operatorname{Ai}\left(|\hat{J}|^{1 / 3} x_{1}\right) \operatorname{Bi}^{\prime}\left(|\hat{J}|^{1 / 3} x_{1}\right)-\operatorname{Bi}\left(|\hat{J}|^{1 / 3} x_{1}\right) \operatorname{Ai}^{\prime}\left(|\hat{J}|^{1 / 3} x_{1}\right)\right)}\right|
\end{aligned}
$$

and the intermediate current-voltage curve is given by

$$
\begin{aligned}
& \Phi+\Phi_{b i}=-2 \log \left|\frac{\hat{J}}{2 \delta}\right| \\
& (3.42) \quad-4 \log \left|\frac{\operatorname{Bi}\left(|\hat{J}|^{1 / 3} x_{1}\right) \operatorname{Ai}\left(|\hat{J}|^{1 / 3}\left(x_{1}-1\right)\right)-\operatorname{Ai}\left(|\hat{J}|^{1 / 3} x_{1}\right) \operatorname{Bi}\left(|\hat{J}|^{1 / 3}\left(x_{1}-1\right)\right)}{|\hat{J}|^{1 / 3}\left(\operatorname{Ai}\left(|\hat{J}|^{1 / 3} x_{1}\right) \operatorname{Bi}^{\prime}\left(|\hat{J}|^{1 / 3} x_{1}\right)-\operatorname{Bi}\left(|\hat{J}|^{1 / 3} x_{1}\right) \operatorname{Ai}^{\prime}\left(|\hat{J}|^{1 / 3} x_{1}\right)\right)}\right|,
\end{aligned}
$$

where $\hat{J}=-J /\left(2 \lambda^{2}\right)$.

3.1.3. The current-voltage curves for small, intermediate, and large $J$. In (3.10), (3.31), and (3.42) we have derived asymptotic expressions for the currentvoltage curve in the limits of small, large, and intermediate currents, respectively. An example of the results obtained using these expressions is plotted in Figure 3.2 for $\Phi_{b i}=4, \delta=e^{-8}, \lambda=1$, and $\theta=0.4$ (where the dashed lines represent the results of the small $J$ asymptotics, the dot-dashed line that of the large $J$ asymptotics, and the solid line that of the intermediate asymptotics). As alluded to previously the small current asymptotics indicates that the current saturates in hard reverse bias with $J \sim 2 \delta /\left(\lambda^{2} \theta\right)$ as $\Phi \rightarrow+\infty$ (see Figure 3.2, right) and that it grows exponentially with $-\Phi$ in hard forward bias with $J \sim-2 \delta \exp (-\Phi / 2)$ as $\Phi \rightarrow+\infty$ (again see Figure 3.2, right). However, for significantly large negative potentials (when $\Phi+\Phi_{b i}$ becomes comparable with $-2 \log (1 / \delta))$ the exponential growth ceases and is replaced by linear growth of the form $J \sim \frac{1}{2}\left(\left(\Phi+\Phi_{b i}\right)+2 \log (1 / \delta)\right) \exp \left(\Phi_{b i} / 2\right)$ for potentials $\Phi+\Phi_{b i}$ significantly less than $-2 \log (1 / \delta)$. This linear growth is shown by the dot-dashed line 
in Figure 3.2, left. Notably there is a mismatch between the asymptotic expressions for the current-voltage curve in the vicinity of $\Phi+\Phi_{b i}=-2 \log (1 / \delta)$ which is explained by the intermediate current asymptotics (solid line).

4. Inclusion of a photo-generated current. Light absorbed in organic photovoltaic diodes produces excitons (strongly bound excited electron-hole pairs) that diffuse through the acceptor and the donor until they either recombine, releasing their energy as heat, or meet the acceptor-donor interface, where they can separate (typically in a multistage process whose details do not concern us here) into a hole in the donor and an electron in the acceptor. We can model this process by including a source term for electrons and holes on the organic-organic interface at $x=0$ replacing the dimensionless equation (2.13) by

$$
\left[\mathcal{F}_{n}\right]_{x=0}=\left[\mathcal{F}_{p}\right]_{x=0}=\frac{J_{\text {phot }}}{q}-R_{b}\left(\left.n\right|_{x=0^{-}},\left.p\right|_{x=0^{+}}\right),
$$

where $J_{\text {phot }}$ is a positive variable representing the contribution to the electric current density from the dissociation of bound excitons at the interface. On nondimensionalizing (as in section 2.4 with $J_{\text {phot }}$ scaling like the current density $J$ ), approximating the electron and hole densities in the donor and acceptor, respectively, by zero (as in section 2.5), and assuming that the diode is antisymmetric (as in section 2.5) leads to a modification to the dimensionless-antisymmetric model $(2.37)-(2.39)$ in which (2.38) is replaced by

$$
J-J_{\text {phot }}=-\frac{2 \delta\left(\left.p^{2}\right|_{x=0}-1\right)}{\left(\lambda^{2} \theta+\left.p\right|_{x=0}\right)} .
$$

Provided $0<J_{\text {phot }} \ll 1$ and $-\Phi \ll \log (1 / \delta)$ the small current asymptotics as expounded in section 3.1 are appropriate. In this regime the leading order solution for the potential is independent of the current density and the leading order current density is derived directly from the solution for the leading order potential. In the modified current equation $J-J_{\text {phot }}$ plays the role of the current density in (2.38). It follows that the leading order current density derived from (4.1) is exactly that derived in section 3.1 with the addition of $J_{\text {phot }}$ so that (3.10) is modified to

$$
J \sim J_{\text {phot }}+4 \delta \frac{\sinh (\Phi / 2)}{1+\lambda^{2} \theta \exp (\Phi / 2)}
$$

5. Comparison to experimental data. As summarized in section 3.1.3, the asymptotic model yields the form of the current at low and high voltages as expressed in (3.10) and (3.31). When a conventional equivalent circuit model is used to model the device, the diode and series resistance behaviors of a cell are characterized by parameters with no physical interpretation. Conversely our model allows us to relate both of these behvaiors to a single set of physical parameters. The novel element to this work therefore is that it relates series resistance and the dark current $J_{0}$ to the same set of parameters. Physically this is because we find that at high voltages current is limited by the resistance of each layer, whereas at low voltages it is limited by the recombination fluxes.

As an example we consider the dark current of a pentacene fullerene bilayer which was recently discussed by Credgington et al. in [4]. Our focus here is not to investigate the physical origin of the recombination current but rather to show how both high and low voltage parts of the $\mathrm{J}-\mathrm{V}$ curve are related. In order to compare our idealized device to real data, it is necessary to account for two effects: shunt 
resistance and series resistance. Comparing experimental data to our model, it is evident that real devices have a much smaller resistance at zero bias than is predicted by our idealized bilayer. In keeping with the literature [4] this behavior is attributed to pinholes which act as short circuits between the two contacts. This can be modeled by adding a constant linear shunt resistance in parallel to our idealized, pinhole-free one-dimensional device. Furthermore, it is important to remove the load resistance from the experimental data before extracting the model parameters. For example, Credgington et al. used a resistor with resistance $R_{\text {load }}=60 \Omega$ to measure the current across the devices. Therefore we removed this contribution to the series resistance by transforming the measured experimental voltage $V_{\exp }$ into the real bias across the device as $V=V_{\text {exp }}-J\left(V_{\text {exp }}\right) R_{\text {load }} A$, where $A$ is the area of the device.

In order to fit real data it is helpful to convert (3.10) and (3.31) into the conventional notation used in equivalent circuit models. At low bias, including the shunt resistance $R_{\text {shunt }}$, the dimensional model takes the form

$$
J_{\text {small }}(V)=\frac{V}{R_{\text {shunt }} A}+J_{0}\left(\exp \left(\frac{V q}{2 k T}\right)-1\right) .
$$

Similarly, at high bias, the dimensional form of the equations becomes

$$
J_{\text {large }}(V)=\frac{\left(V-V_{0}\right)}{A R_{\text {series }}} .
$$

The parameters of these equivalent circuit models can be related to the parameters of our model as follows:

$$
\begin{aligned}
J_{0} & =2 J_{D} \delta \\
V_{0} & =\Phi_{b i}+\frac{2 k T}{q} \log \left(\frac{1}{\delta}\right), \\
A R_{\text {series }} & =\frac{2 k T}{J_{D} q} \exp \left(\frac{-q \Phi_{b i}}{2 k T}\right)=\frac{2 k T}{q J_{D} \delta} \exp \left(\frac{-q V_{0}}{2 k T}\right),
\end{aligned}
$$

where we have introduced the notation $J_{D}=q \frac{\bar{D} \Pi_{0}}{L}$ for the diffusion current density used to nondimensionalize currents. Note that (5.4) and (5.5) only allow us to determine the combination $J_{D} \delta$, whereas (5.5) does not allow us to find either $\Phi_{b i}$ or $\delta$ independently. The main observation we make is that $J_{D} \delta$ can therefore be determined experimentally in two independent ways: either by measuring $J_{0}$ or by measuring $R_{\text {series }}$ and $V_{0}$.

Fitting the data using these two methods gives values of $J_{D} \delta=7.2 \times 10^{-3} \mathrm{Am}^{-2}$ and $J_{D} \delta=2.8 \times 10^{-3} \mathrm{Am}^{-2}$ using the low and high bias formulae, respectively. These parameters were obtained by applying a least squares fit to (5.1) and (5.2) using data only at extreme ends of the voltage range. Figure 5.1 shows a good fit for such models and lists the values found. Taking values for the electron diffusivity in fullerene of $6.3 \times 10^{-9} \mathrm{~m}^{2} \mathrm{~s}^{-1}$ and assuming an intrinsic carrier density $\Pi_{0}$ of $2.5 \times 10^{25} \mathrm{~m}^{-3}$ [15], we find that $\delta=O\left(5 \times 10^{-9}\right)$, which justifies our asymptotic treatment of the problem. Of course, the value of $J_{D} \delta$ could be obtained using more sophisticated techniques, for example, by numerically simulating the entire $\mathrm{J}-\mathrm{V}$ curve. However, our main result is that the two values found are very similar and hence that extremes of the curve can be fitted consistently with a single model. In addition, we note that we developed the model for a highly simiplified case, assuming the device to be symmetric and using $\mathrm{SRH}$ recombination via a single trap level (so that the ideality factor is necessarily 


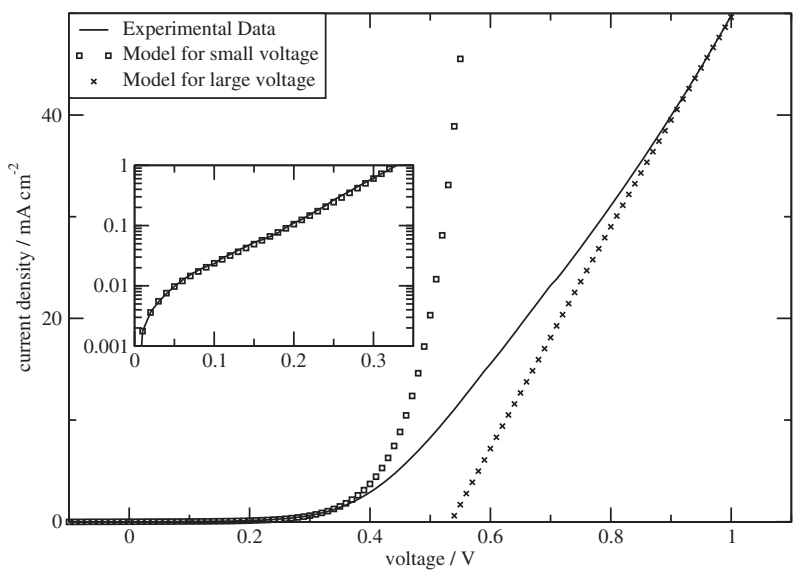

FIG. 5.1. Experimental (line) and modeled (symbols) dark current characteristic for a fullerenepentacene bilayer device. The data shows the logarithm of the current density. The experimental curves were fitted using (5.1) and (5.2) with parameters $R_{\text {shunt }}=153 \mathrm{k} \Omega, J_{0}=1.4310^{-6} \mathrm{Acm}^{-2}$, $V_{0}=0.506 \mathrm{~V}, R_{\text {series }}=160 \Omega$. The device parameters were $R_{\text {load }}=60 \Omega, A=0.045 \mathrm{~cm}^{2}$, and finally $\frac{k T}{q}=25 \mathrm{mV}$.

2). These simplifications could be removed, the analysis repeated, and more accurate physical parameters determined. However, it is important to note that an excellent fit has been obtained without such complications, indicating that the essence of the physical process has been captured in the model.

6. Discussion and conclusions. The asymptotic analysis presented in this paper is successful in predicting analytic relationships for the current-voltage curve of a flat organic semiconducting diode. The curve has three distinct parts: the low-current part is characteristic of a conventional diode showing current saturation in reverse bias and exponential behavior in forward bias; the high-current part is dominated by the Ohmic resistance of the bulk, usually interpreted as a series resistance; and there is a subtle intermediate-current behavior that covers a relatively small range of potentials and currents and which smoothly connects the other two parts. The current-voltage curve displays the same features as the phenomenological Shockley equivalent circuit model with small shunt resistance (see, for example, [19]). That is, in reverse bias, it saturates to a constant current, in forward bias it displays exponential behavior, and for very large forward bias the behavior is linear. As shown in [19] such models can be very successfully fitted to experimental data. For the simple model presented here the ideality factor is always 2 . This is a consequence of the recombination law assumed at the interface, which is in our case proportional to charge density. Recombination laws with a different dependence on charge density could be exploited to model different ideality factors.

The diode can act as a photovoltaic device when exposed to radiation of the right wavelengths. This leads to the generation of excitons states (strongly bound electronhole pairs) which, if they reach the acceptor-donor interface before recombining, can separate into an electron in the acceptor and a hole in the donor and drive an electric current. Typically organic photovoltaics operate in the low-current limit, in which the device lies close to its equilibrium. In this limit the additional photo-generated current $J_{g}$ has the effect of shifting the current-voltage curve (as plotted in the right panel of Figure 3.2) up the $J$-axis by an amount $J_{\text {phot }}$. 
The analysis presented has assumed that the Poole-Frenkel effect is negligible. Notably this effect does not change the small current behavior since the system is in quasi equilibrium away from the interface. The Poole-Frenkel term does, however, change the position of the intermediate region of the current-voltage curve and in particular delays the transition to the high-current (and intermediate) limits since it enhances diffusion at high electric fields, thus keeping the system near quasi equilibrium for longer. In the high-current limit the nonlinear resistance encapsulated in the Poole-Frenkel effect leads to a nonlinear current-voltage curve that in the limit of large (negative) applied voltage $\Phi$ has asymptotic behavior that depends upon the Poole-Frenkel parameter $\nu$ through

$$
\log (-J) \sim \nu\left(-\frac{\Phi+\Phi_{b i}}{2}\right)^{1 / 2}+\frac{\Phi_{b i}}{2}
$$

In fact our analysis points to the interesting observation that it may be possible to use the series resistance part of the current-voltage curve to better understand transport in the bulk of the semiconductors.

The analysis performed here forms the basis of an understanding of the behavior of organic photovoltaic devices. The effects of illumination of the photovoltaic device have been considered where the photovoltaic generation is relatively small and could be treated more generally together with a description of the behavior of the generation and transport of excitons, as well as their dissociation, into electrons and holes at the interface. In practice the interface between the donor and acceptor in a photovoltaic device is rarely planar and is typically manufactured with a highly convoluted structure in order to maximize the chance of excitons diffusing there. For such devices we believe that an approach based on homogenizing the drift diffusion equations in acceptor and donor over the scale of the microstructure may be more appropriate. Extending the analysis to account for these and other phenomena remains an open question.

Acknowledgments. The authors would like to thank Savina Joseph for her help in preparing the manuscript. Special thanks go to Dr. Credgington and Prof. Durrant for allowing us access to their original data.

\section{REFERENCES}

[1] J.A. Barker, C.H. Ramsdale, and N.C. Greenham, Computer simulation of polymer solar cells, Model. Simul. Mater. Sci. Eng., 15 (2007), pp. 13-26.

[2] D. Brinkman, K. Fellner, P.A. Markowich, and M.-T. Wolfram, A drift-diffusionreaction model for excitonic photovoltaic bilayers: Asymptotic analysis and a 2D HDG finite-element scheme, Math. Model. Methods Appl. Sci., to appear, DOI:10.1142/S0218202512500625.

[3] G.A. Buxton and M. Clarke, Modelling the current-voltage characteristics of bilayer polymer photovoltaic devices, Phys. Rev. B, 67 (2003), 075205.

[4] D. Credgington, Y. Kim, J. Labram, T.D. Anthopoulos, and J. Durrant, Analysis of recombination losses in a pentacene/C60 solar cell, J. Phys. Chem. Lett., 2 (2011), pp. 27592763.

[5] B.K. Crone, P.S. Davids, I.H. Campbell, and D.L. Smith, Device model investigation of bilayer organic light emitting diodes, J. Appl. Phys., 87 (2000), pp. 1974-1982.

[6] P.M. Borsenberger and D. Weiss, Organic Photoreceptors for Xerography, Marcel Dekker, New York, 1998.

[7] P.S. Davids, I.H. Campbell, And D.L. Smith, Device model for single carrier organic diodes, J. Appl. Phys., 82 (1997), pp. 6319-6325. 
[8] C. De Falco, R. SACCO, ANd M. Verri, Analytical and numerical study of photocurrent transients in organic polymer solar cells, Comput. Methods Appl. Mech. Engrg., 199 (2010), pp. $1722-1732$.

[9] B. Geoffrey, P. Le Roy, And C. Prat, Organic light-emitting diode (OLED) technology: Materials, devices and display technologies, Pol. Int., 55 (2006), pp. 572-582.

[10] S. Günes, H. Neugebauer, And N.S. SARiCiftCi, Conjugated polymer-based organic solar cells, Chemical Rev., 103 (2007), pp. 1324-1338.

[11] K.A. Gregg and M.C. Hanna, Comparing organic to inorganic photovoltaic cells: Theory, experiment, and simulation, J. Appl. Phys., 93 (2003), pp. 3605-3614.

[12] P.E. De Jongh and D. Vanmaekelbergh, Trap-limited transport in assemblies of nanometersize TiO2 particles, Phys. Rev. Lett., 77 (1996), pp. 3427-3430.

[13] T. Kirchartz, B.E. Pieters, J. Kirkpatrick, U. Rau, and J. Nelson, Recombination via tail states in polythiophene: Fullerene solar cells, Phys. Rev. B, 83 (2011), 115209.

[14] J. Kirkpatrick, V. Marcon, K. Kremer, J. Nelson, and D. Andrienko, Charge mobility in discotic mesophases: A multiscale quantum and classical study, Phys. Rev. Lett., 98 (2007), 227402.

[15] L.J.A. Koster, E.C.P. Smits, V.D. Minailetchi, and P.W.M. Blom, Device model for the operation of polymer/fullerene bulk heterojunction solar cells, Phys. Rev. B, 72 (2005), 085205.

[16] C.M. Martin, V.M. Burlakov, H.E. Assender, and D.A.R. Barkhouse, A numerical model for explaining the role of the interface morphology in composite solar cells, J. Appl. Phys., 102 (2007), 104506.

[17] J. Nelson, The Physics of Solar Cells, Imperial College Press, London, 2003.

[18] L. Pautmeier, R. Richert, And H. BÄssler, Poole-Frenkel behaviour of Charge Transport in organic solids with off-diagonal disorder studied by Monte Carlo simulation, Synth. Methods, 37 (1990), pp. 271-281.

[19] W.J. Potscavage, S. Yoo, and B. Kippelen, Origin of the open-circuit voltage in a multillayer heterojunction organic solar cells, Appl. Phys. Lett., 93 (2008), 193308.

[20] Y. Seunhyup, W.J. Potscavage, B. Domercqua, T.D. Lic, S.C. Jones, R. Szozskiewicz, D. Levib, E. Riedoc, S.R. Marder, and B. Killen, Analysis of improved photovoltaic properties of pentacene/C60 organic solarcells: Effects of exciton blocking layer thickness and thermal annealing, Solid-State Electron., 51 (2007), pp. 1367-1375.

[21] B. StuART, Solarmer achieves 8.13 percent OPV efficiency, PV Magazine, July, 2010.

[22] B. StuART, Heliatek achieves 10.7 percent OPV efficiency; to begin module production this year, PV Magazine, April, 2012.

[23] S.M. Sze And K. NG Kwok, Physics of Semiconductor Devices, 3rd ed., Wiley-Interscience, New York, 2006.

[24] C. Tansae, P.W.H. Blom, D.M. De Leeuw, and E.J. Meijer, Charge carrier density dependence of mobility in poly(p-phenylene vinylene), Phys. Stat. Sol. B, 201 (2004), pp. 12361245.

[25] S.W. Thomas, III, G.D. Joly, And T.M. Swager, Chemical sensors based on amplifying fluorescent conjugated polymers, Chem. Rev., 107 (2007), pp. 1339-1386.

[26] J. Zaumseil AND H. SiRRinghaus, Electron and ambipolar transort in organic field-effect transistors, Chem. Rev., 107 (2007), pp. 1296-1323. 